\title{
Tagged electron neutrinos
}

\section{A. Longhin}

I.N.F.N., Laboratori Nazionali di Frascati, Frascati (Rome), Italy

E-mail: andrea.longhin@lnf.infn.it

\section{Ludovici}

I.N.F.N., Sezione di Roma, Rome, Italy

E-mail: lucio.ludovici@romal.infn.it

\section{F. Terranova*}

Univ. of Milano-Bicocca and I.N.F.N. sez. Milano-Bicocca

E-mail: francesco.terranova@mib.infn.it

\begin{abstract}
The advantages of identifying charged leptons in the decay tunnel of conventional neutrino beams are known since long but the technologies that can sustain the corresponding rates and doses became available only recently. We revise these technologies demostrating that a very precise monitoring of $v_{e}$ at source can be achieved tagging the positrons in $K^{+} \rightarrow e^{+} \pi^{0} v_{e}$ decays. We discuss facilities where positron monitoring is employed to reduce the uncertainties on the $v_{e}$ flux and improve substantially our knowledge of cross sections ("single tag mode"). We also consider more ambitious setups where the time correlation between lepton and $v \mathrm{CC}$ event at the far detector is exploited ("event by event tag mode").
\end{abstract}

16th International Workshop on Neutrino Factories and Future Neutrino Beam Facilities - NUFACT2014, 25 -30 August, 2014

University of Glasgow, United Kingdom

${ }^{*}$ Speaker. 


\section{Introduction}

Conventional neutrino beams employ charged particle monitoring at source to constrain the neutrino flux. At present, both the primary protons (protons-on-target, pot) and the muons at the beam dump are monitored. The former provide the integral neutrino flux once the $v$ /pot ratio is known; the latter is used to estimate flux variations during data taking and beam misalignment. The time coincidence between the proton bunch extraction and the neutrino events at the far detector is also employed to reject cosmic ray background.

The experiments, however, are not able to correlate the neutrino CC interaction at the far detector with the corresponding lepton at the neutrino production vertex in the decay tunnel ("tagged neutrino beams" [1]) due to the high particle rate before the beam dump. Similarly, the $v_{e}$ and $v_{\mu}$ flux at source is indirectly estimated by the integrated pot. The $v /$ pot ratio is computed from hadroproduction data and a full simulation of particle transport and decay up to the beam dump. As a consequence, all neutrino cross section measurements are intrinsically limited to $\sim 10 \%$ by flux uncertainties. For the same reason, the level of purity and/or the knowledge of the contamination of conventional beams cannot achieve what is envisaged at the Neutrino Factory (or Beta Beam) where there is a straightforward link between the number of stored muons (ions) and the neutrino flux at source. This uncertainty can be substantially reduced if:

- the decays producing $v_{e}$ in the beam can be monitored measuring the corresponding positron rate in the decay tunnel ("single tag mode")

- the observed neutrino events can be time-correlated with the positron in the decay tunnel ("event by event tag mode")

Single tag mode is particularly effective to reduce flux systematics in cross section measurements [2] and lower the overall uncertainty down to $\sim 1 \%$ [3]. It is a technique that uses conventional neutrino beams and detector technologies developed for HEP colliders (LHC, ILC, CLIC). It can also be extended (see Sec. 2 below) to measure the $v_{\mu}$ cross section with a comparable precision.

The "event by event tag mode" is a modern implementation of the tagged neutrino beam concept. It can be used to veto on an event by event basis the $v_{e}$ contamination in short baseline experiments [4] and measure the neutrino energy from the kinematics of the parent decay but prevents the use of horns to focus secondaries and is challenged by cosmic ray background due to the longer proton extraction. This operation mode is discussed in Sec. 3.

\section{Single tag mode}

Single tag facilities can be built from $v_{\mu}$ neutrino beams where the contamination of $v_{e}$ is mostly due to three body $K^{+}$decays $\left(K_{e 3}\right.$, i.e. $\left.K^{+} \rightarrow e^{+} \pi^{0} v_{e}\right)$. The positrons are observed in the decay tunnel by purely calorimetric techniques and the rate can be reduced to $<1 \mathrm{MHz} / \mathrm{cm}^{2}$ since the positrons and all other particles from $K^{+}$decays are produced at angles significantly larger that muons from two-body decay of $\pi^{+}$. The beam-line can be optimized to increase the $v_{e}$ components from $K_{e 3}$ and suppress to a negligible level the $v_{e}$ contamination from muon decays. 


\begin{tabular}{cccc}
\hline \hline Channel & $v$ at detector & Angular spread & \\
\hline$\pi^{+} \rightarrow \mu^{+} v_{\mu}$ & Bulk of $v_{\mu}$ & 4 mrad for $\mu^{+}$ & 2-body decay \\
$\pi^{+} \rightarrow \mu^{+} v_{\mu} \rightarrow e^{+} v_{e} \bar{v}_{\mu} v_{\mu}$ & (DIF) $v_{e}+\bar{v}_{\mu}$ & $28 \mathrm{mrad}$ for $e^{+}$ & $\begin{array}{c}\text { 3-body decay } \\
\text { (low parent mass) }\end{array}$ \\
$K^{+} \rightarrow e^{+} v_{e} \pi^{0}$ & $v_{e}$ from $K_{e 3}$ & $88 \mathrm{mrad}$ for $e^{+}$ & $\begin{array}{c}\text { 3-body decay } \\
\text { (high parent mass) }\end{array}$ \\
Undecayed $K^{+}, \pi^{+}, \mathrm{p}$ & none & $3 \mathrm{mrad}$ & no prompt positron \\
Other $K^{+}$decays & $v_{\mu}$ or none & & negligible \\
Wrong sign and off momentum & & &
\end{tabular}

Table 1: Particles in the decay tunnel, angular spread and contributions to the neutrino flux at the detector.

A specific configuration aimed at measuring the $v_{e}$ cross section at the per cent level is discussed in Ref. [3]. In that case, protons extracted from an accelerator in 2 ms spills impinge on a Be target and produce secondary hadrons that are captured, sign selected and transported to the instrumented decay tunnel. The tunnel is short $(50 \mathrm{~m})$ compared with the decay length of the transported pions ( $476 \mathrm{~m}$ at $8.5 \mathrm{GeV}$ ). The reduction of flux, however, is compensated by the significant increase of the $v_{e} / v_{\mu}$ ratio $(\sim 2 \%)$ and a nearly complete removal of the contamination of $v_{e}$ from the decay in flight of muons (DIF).

The focusing and transport system selects $\pi^{+}$and $K^{+}$that enter the tunnel in a $10 \times 10 \mathrm{~cm}^{2}$ window in the transverse plane with a flat polar angle distribution (up to $3 \mathrm{mrad}$ ). The particles in the decay tunnel and their angular spread are summarized in Table 1.

The walls of the decay tunnels are fully instrumented with a calorimeter that measures the $e^{+}$ energy and performs $\pi^{+} / e^{+}$separation, and a " $t_{0}$ layer" acting as pre-shower for photon rejection. Technologies that can achieve high efficiency and purity at sustainable costs - $\mathscr{O}(20 \mathrm{M} \$)$ - are discussed in [3]. For sake of reference, we consider here a copper-plastic scintillator Shashlik calorimeter with three longitudinal samples $\left(5 X_{0}, 10 X_{0}\right.$ and $\left.\sim 3 \lambda\right)$. The light is collected by WLS fibers running at $88 \mathrm{mrad}$ with respect to the longitudinal axis of the decay tunnel, i.e. at the mean angle of positrons from $K_{e 3}$. The fibers are optically linked to SiPM. In this configuration, for a hollow cylinder calorimeter with $40 \mathrm{~cm}$ inner radius and $57 \mathrm{~cm}$ outer radius, the $K_{e 3}$ positrons and most of the background particles from hadronic decays of $K^{+}$cross up to $3 \mathrm{Cu}$ interaction lengths $(\lambda=15 \mathrm{~cm})$. The $t_{0}$ layer, based on plastic scintillators or large surface low gain APD's, can be located either inside the vacuum tank of the decay tunnel or in front of the calorimeter. In both cases, the background is dominated by $\pi^{+} \rightarrow e^{+}$misidentification. The efficiencies are $\sim 60 \%$ $(40 \%)$ for a purity level of $18 \%(8 \%)$.

Particles that do not decay in the tunnel $\left(\pi^{+}, K^{+}, p\right)$ and all muons from the 2-body decay of $\pi^{+}$will reach the dump without crossing the $t_{0}$ layer and the calorimeter. The overall rate at the calorimeter will hence be several order of magnitudes smaller than the pion and muon rate at the beam dump and it will be dominated by kaon decays. For a $2 \mathrm{~ms}$ proton extraction and $10^{10}$ pions per spill at the entrance of the decay tunnel, these rates never exceed $500 \mathrm{kHz} / \mathrm{cm}^{2}(5 \mathrm{MHz}$ per tile if the calorimeter has a granularity of $10 \mathrm{~cm}^{2}$ ). The rates for each type of particles crossing the $t_{0}$ layer are summarized in Table 2, while the rates as a function of extraction time are shown in 


\begin{tabular}{cc}
\hline \hline Particle & $\begin{array}{c}\text { Maximum rate } \\
\left(\mathrm{kHz} / \mathrm{cm}^{2}\right)\end{array}$ \\
\hline$\mu^{+}$ & 190 \\
$\gamma$ & 190 \\
$\pi^{+}$ & 100 \\
$e^{+}$ & 15 \\
all & 495 \\
\hline \hline
\end{tabular}

Table 2: Particle rates at the $t_{0}$ layer and calorimeter.

Fig. 1.

Since in single tag mode, no time coincidence is requested between the lepton and the neutrino interaction at the far detector, constraints on the timing resolution of the $t_{0}$ layer and on the proton extraction length are loose. In particular, single tag facilities can employ standard magnetic horns, which in turn enhance significantly the neutrino flux at the far detector. In [3] it is shown that a single tag facility complemented by a 500 tons $v_{e}$ detector located $100 \mathrm{~m}$ from the entrance of the decay tunnel can measure the total $v_{e}$ cross section at the per cent level $(20 \times$ improvement with respect to current measurement [5]) in about 1 year, i.e. with $5 \times 10^{19}$ pot at the CERN-SPS or $1.2 \times 10^{20}$ pot at the Fermilab Main Injector.

In addition, the radial distribution of neutrinos in the detector strongly depends on the parent particle. $v_{\mu}$ neutrinos from pion decays cluster at radii lower than $\sim 2 \mathrm{~m}$, while $v_{\mu}$ from $K^{+} \rightarrow$ $\mu^{+} v_{\mu}$ and $K^{+} \rightarrow \pi^{0} \mu^{+} v_{\mu}$ extend to larger radii. The $v_{\mu}$ CC sample at large radii (from $K^{+}$decays) can thus be normalized by the large angle muons rate at the calorimeter or, indirectly, by the $K^{+}$ yield estimated from the positron rate. This technique ("space correlation") can be employed to improve the flux systematic uncertainty in $v_{\mu} \mathrm{CC}$ cross section measurements.

Finally, it is worth mentioning that $\bar{v}_{e}$ cross sections can be measured running in inverse polarity mode without significant changes in efficiency and purity. The statistics is, however reduced by the difference in the kaon yield at the target and by the smaller anti-neutrino cross section.

\section{Tagging on an event by event basis}

In spite of early proposals [1] and experimental attempts performed in the 80's [6], an event by event association between the neutrino interaction at the detector and the corresponding lepton in the decay tunnel has never been observed. This originates from the fact that the ratio between the number of kaons at source and the number of observed $v_{e}$ is extremely large: $\mathscr{O}\left(10^{13}\right)$ kaons per $v_{e} \mathrm{CC}$ event. Since the overall kaon decay rate along the tunnel must be small to time tag the particles, the integrated amount of kaons is not sufficient to reach a significant statistics at the neutrino detector. This constraint can be relieved improving the time resolution of the detectors and increasing the duty cycle of the accelerator for long extraction times.

The intrinsic limit to time resolution is due to the finite emission angle of the positron, which introduces an uncertainty on the time tagging of the order of a few tens of ps [3, 4]. In fact, time resolution of neutrino detectors limits the tagging precision to $\sim 1 \mathrm{~ns}$, i.e. well above the intrinsic 


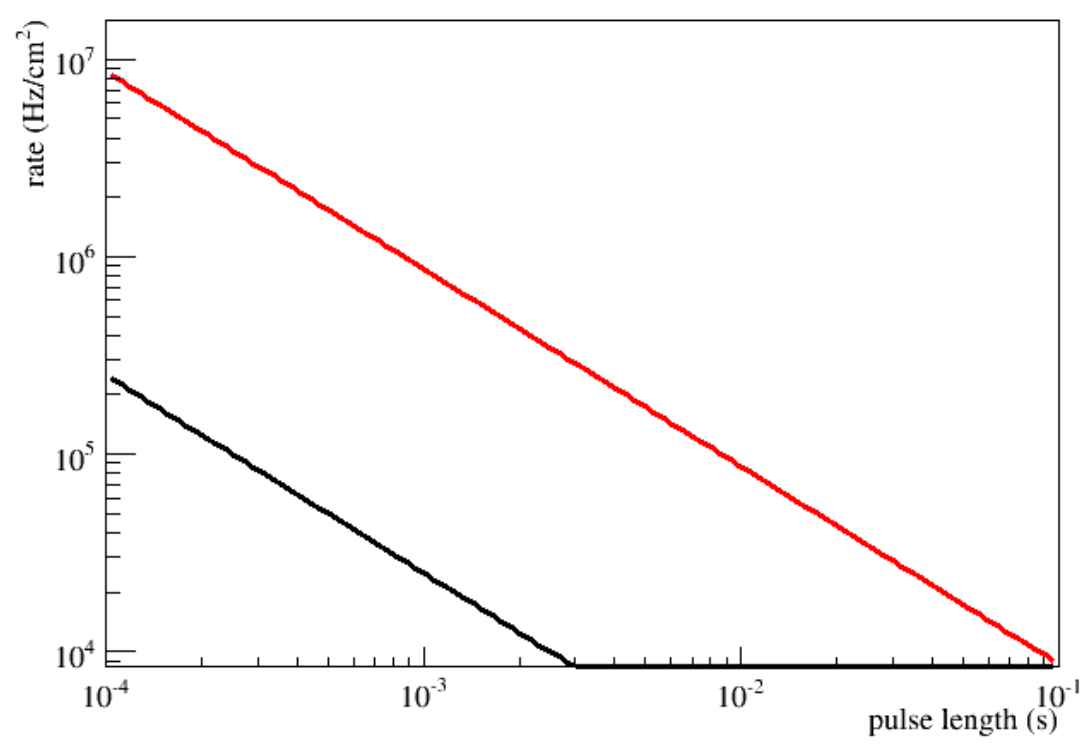

Figure 1: Total particle rate (red line) and positron rate (black line) as a function of the proton extraction length.

limit from positron emission. Fig. 2 shows the probability of a false tag in the $\Delta t-T_{\text {extr }}$ plane, where $\Delta t$ is the linear sum of the time resolution of the $t_{0}$ layer and the neutrino detector, and $T_{\text {extr }}$ is the proton extraction length (both in s).

An upgrade of a single tag facility to event by event tag is thus conceivable if $T_{\text {extr }} \simeq 1 \mathrm{~s}$. These extraction times have been reached at low intensity in Protvino and are currently considered at CERN-SPS for the high intensity beam dump experiment SHiP [7]. In fact, a proof-of-principle of tagging on an event by event basis, i.e. a simultaneous measurement of a $v_{e} \mathrm{CC}$ and the corresponding positron at the decay tunnel is within reach of current technologies. On the other hand, a concrete exploitation of this operation mode for the aims mentioned in Sec. 1 requires additional R\&D. In particular, two challenges have to be addressed [3]:

- Long extraction times prevent the use of magnetic horns. Dipole+quadrupole focusing systems can replace the horns but this comes at the expense of a $\sim 8 \times$ reduction of the flux due to their smaller acceptance (see Table 2 of [3]).

- A $1 \mathrm{~s}$ extraction time combined with detector resolution of $1 \mathrm{~ns}$ increases the background contamination due to cosmics by one order of magnitude.

In addition, if the neutrino energy has to be determined by kinematic reconstruction of the kaon decay products, the momentum bite of the focusing system cannot exceed $\sim 10 \%$. In this case, the $v_{e}$ energy resolution will be limited by the calorimeter to about $15 \%$ for $3 \mathrm{GeV}$ neutrinos.

\section{Conclusions}

The measurement of the large angle positrons in conventional neutrino beams offers unprece- 


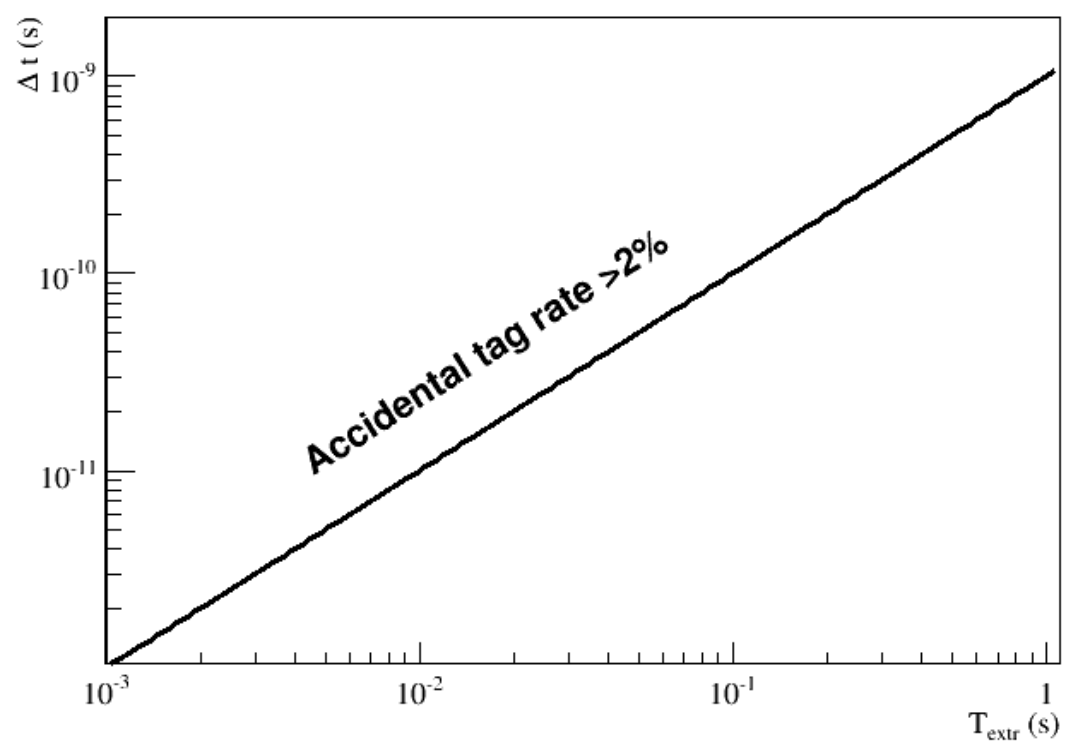

Figure 2: Time resolution $\left(\Delta t\right.$ - see text) versus proton extraction length $\left(T_{\text {extr }}\right)$. The area above the line corresponds to a rate of accidental tags greater than $2 \%$.

dented opportunities to improve our knowledge of cross sections, well below the intrinsic limit due to flux uncertainties. The positron rate can be exploited in a "single tag facility" that takes advantage of conventional focusing horns and detector technologies developed at colliders. This facility can be upgraded to run in slow extraction mode and perform for the first time an event by event neutrino tagging, i.e. observe simultaneously the $v_{e}$ at the detector and the corresponding positron in the decay tunnel. Still, the exploitation of event by event tagging for high statistics experiments remains challenged by the small acceptance of static focusing systems and by cosmic background reduction.

\section{References}

[1] L. N. Hand, A study of 40-90 GeV neutrino interactions using a tagged neutrino beam, Proceedings of Second NAL Summer Study, Aspen, Colorado, 9 Jun - 3 Aug 1969, p.37; B. Pontecorvo, Tagging direct neutrinos. A first step to Neutrino Tagging, Lett. Nuovo Cim. 25 (1979) 257; S. P. Denisov, S. A. Mukhin, A. A. Spiridonov and V. P. Zhigunov, Calculation of tagged neutrino beams for Unk, (in Russian) preprint IHEP 81-98, Serpukhov, 1981; R.H. Bernstein and F. Borcherding, A new method of measuring $\sin ^{2} \theta_{W}$ in Deep Inelastic Scattering, FERMILAB-Proposal-0788, 1989.

[2] L. Ludovici and F. Terranova, Electron neutrino tagging through tertiary lepton detection, Eur. Phys. J. C 69 (2010) 331 [arXiv: 1004.2904 [hep-ex] ].

[3] A. Longhin, L. Ludovici and F. Terranova, A novel technique for the measurement of the electron neutrino cross section, arXiv:1412.5987 [hep-ex] .

[4] L. Ludovici and P. Zucchelli, Conceptual study of an 'antitagged' experiment searching for $v_{\mu} \rightarrow v_{e}$ oscillation, hep-ex/9701007. 
[5] K. Abe et al. [T2K Collaboration], Measurement of the inclusive electron neutrino charged current cross section on Carbon with the T2K Near Detector, Phys. Rev. Lett. 113 (2014) 241803 [arXiv:1407.7389 [hep-ex]].

[6] V. V. Ammosov et al., Experiments with tagged neutrino beam at IHEP accelerator ,Proposal SERP-E-152, Serpukhov, 1984; G. Bohm, Project of a Tagged Neutrino Facility at Serpukhov, Proceedins of the X Warsaw Symposium on elementary particle physics (Z. Ajduk ed.), Kazimierz, Poland, May 24-30, 1987.

[7] G. Arduini et al., A new Experiment to Search for Hidden Particles (SHIP) at the SPS North Area, Preliminary Project and Cost Estimate, CERN EN-DH-2014-007, 2014. Available at http://ship.web.cern.ch/ship/ 